\title{
Der Verbund Handschriften, Archive, Nachlässe (HAN) gestern, heute, morgen
}

\begin{abstract}
Im Aleph-Verbundkatalog HAN (Handschriften, Archive, Nachlässe) katalogisieren heute elf schweizerische Bibliotheken und Archive ihre Handschriften- und Archivbestände. Im von der Universitätsbibliothek Basel betriebenen Katalog sind zurzeit knapp 300’000 Dokumente von der Spätantike bis zur Gegenwart erfasst. Der vorliegende Artikel zeichnet die gut zehnjährige Geschichte des Verbunds nach, beschreibt seine heutige Organisationsstruktur, bewertet Vor- und Nachteile des Katalogs und geht auf einige der Fragen ein, die sich mit Blick auf die Zukunft, d. h. konkret auf die Einführung von SLSP, stellen.
\end{abstract}

Das Akronym HAN steht für vieles, wie ein Blick auf die entsprechende Übersichtsseite in der Wikipedia lehrt. Immerhin zeigt mir Google unseren Verbundkatalog HAN gleich nach ,Han - Mongolian Barbecue‘ an zweiter Stelle an. Diese Restaurantkette war uns mit der Registrierung der Adresse www.han.ch leider zuvor gekommen. ${ }^{1}$ Der heutige Verbund HAN hatte im Laufe seiner Geschichte verschiedene Namen und das Akronym wurde nicht immer gleich aufgelöst. Es bringt zum Ausdruck, dass dieser Katalog dafür konzipiert wurde, alle Materialien, die in den Bibliotheken traditionellerweise in den Sondersammlungen aufbewahrt werden, zu erschliessen und zu verwalten: Handschriften, Briefe, Autographen, Archivalien, Bilder etc. Im Namen konnten aus praktischen Gründen nicht alle Dokumententypen aufgenommen werden, weshalb man sich auf die Haupttypen Handschriften im umfassenden Sinn und Archivbestände beschränkte. Da Letztere in Bibliotheken meist in Form von Nachlässen und unter diesem Namen vorhanden sind, wurden sie speziell hervorgehoben. Dass die alten Drucke, Karten und Graphiken in der Regel in den Hauptkatalogen nachgewiesen werden, ergab sich aus der historischen Entwicklung.

1 Heute findet man den Katalog HAN unter http://aleph.unibas.ch/F/?func=option-update-lng\& file_name=find-b\&p_con_lng=GER\&local_base $=$ dsv05 und die Webseite zum Verbund unter htt p://www.ub.unibas.ch/han/. Alle Links in diesem Beitrag wurden am 26.03.2018 überprüft.

Danksagung: Ich danke Barbara Gygli Dill, Rudolf Gamper und Beat Mattmann für ihre kritische Lektüre dieses Artikels und ihre Verbesserungsvorschläge.

Ә Open Access. () Ueli Dill, publiziert von De Gruyter. (c) BY-NC-ND Dieses Werk ist lizenziert unter der Creative Commons Attribution-NonCommercial-NoDerivatives 4.0 Lizenz. 


\section{Vorgeschichte}

Als sich in den 1990er Jahren der Informationsverbund Deutschschweiz (IDS) zu formieren begann und ab 1999 die ersten Aleph-Installationen produktiv wurden, stellte sich an der Universitätsbibliothek Basel die Frage, wie man mit den bis dahin in der Datenbank Ledoc erfassten Metadaten zu den Nachlässen verfahren solle. Die Grundfrage, ob man sie in den Hauptkatalog importiere ober ob eine weitere Aleph-Datenbank nötig sei, wurde zugunsten der zweiten Lösung entschieden.

Pierre Gavin konzipierte in der Folge DSV05 (bibliographische Daten) und DSV15 (Normdaten), die unter anderen Namen in weiteren Bibliotheken (ZHB Luzern: ILU05, KB Vadiana St. Gallen: SGB05, Rorschach-Archiv, Gosteli-Archiv u.a.) zum Einsatz kamen. Gavin steht damit am Anfang der Geschichte von HAN und er begleitete das Projekt in seinen Aufbaujahren noch länger, indem er das Format immer wieder an die neuen Anforderungen anpasste und dank seiner grossen Erfahrung die Entwicklung mit wertvollen Ratschlägen steuerte. Er richtete sich bei der Festlegung der zu verwendenden MARC-Felder nach Encoded Archival Description (EAD), dem XML-Standard für archivische Findemittel. Damit war eine gestufte archivische Erschliessung im vorhandenen Bibliothekssystem möglich. Allerdings wurden, zumindest in Basel, die Möglichkeiten der neuen Installation nicht voll ausgeschöpft.

Im Herbst 2005 entschlossen wir uns deshalb in der UB Basel dazu, dieses schlummernde Potenzial besser auszunützen und, darüber hinausgehend, $z u$ versuchen, alle Sondermaterialien, die nicht schon im Hauptkatalog erfasst wurden, d.h. vor allem mittelalterliche und neuzeitliche Handschriften, ebenfalls in DSV05 unterzubringen. Bei dieser Gelegenheit wurde die Frage, ob die Sonderbestände nicht im Hauptkatalog erfasst werden könnten bzw. sollten, noch einmal gründlich diskutiert. Zwar blieb ein einziger Katalog das längerfristige Ziel, doch wäre der Weg dazu damals so schwierig und zeitaufwendig gewesen, dass wir beschlossen, zuerst einmal DSV05 auszubauen.

Die Grundprinzipien, die wir bei dieser Arbeit befolgten, waren:

- Wir benutzen eine einzige Datenbank für alle historischen Sondermaterialien mit der Option einer späteren Fusion mit dem Hauptkatalog.

- Das Format soll den Anforderungen der historischen Materialien entsprechen, sich aber möglichst eng an IDS-MARC orientieren bzw. heute nach der Umstellung des IDS auf RDA an MARC21. Konkordanzen zu fachspezifischen Formaten und Standards wie Encoded Archival Description (EAD) oder Text Encoding Initiative (TEI) sollen die Interoperabilität mit entsprechend aufgebauten Katalogen sicherstellen.

- Das Regelwerk soll einerseits bestehende fachspezifische Regelwerke bzw. Richtlinien integrieren, wie z.B. Descriptive Cataloging of Ancient, Medieval, 
Renaissance, and Early Modern Manuscripts (AMREMM), ISAD(G) oder die Regeln zur Erschließung von Nachlässen und Autographen (RNA), in der Grundanlage sich aber eng an die Katalogisierungsregeln IDS (KIDS) anlehnen. Dies soll es ermöglichen, Mitarbeitende, welche über eine ,normale‘ Katalogausbildung verfügen, möglichst leicht zu schulen und einzusetzen.

Im April 2006 berichtete ich erstmals auf der Jahrestagung der Handschriftengruppe des BIS, bzw. damals noch BBS, von den Basler Plänen und erhielt von manchen Kollegen positive Rückmeldungen. Im Juni 2006 übernahm Pierre Gavin die Aufgabe, das Format der Datenbank DSV05, das bereits ISAD(G)-tauglich war, auch an die Anforderungen von AMREMM zu adaptieren. Im August stellte er das Projekt in Zürich auf der DACH-Tagung der Ex Libris-Anwender (DACHELA) vor. In einem weiteren Vortrag präsentierte Andres von Arx seine Lösung, wie die Katalogdaten aus der Nachlasserschliessung hierarchisch in der Art eines Archivdatenplans dargestellt werden können. Im Dezember 2006 begann Mikkel Mangold mit der Übersetzung und Adaption von AMREMM, Pierre Gavin arbeitete an der zehnten Version des Formats von DSV05.

Ungefähr zur selben Zeit entschloss sich die Zentralbibliothek (ZB) Zürich, ihre bisher in einer Allegro-Datenbank erfassten Briefbestände in Aleph, konkret in der Datenbank EBI01, zu katalogisieren, und nahm Kontakt mit uns auf. Am 12. Dezember 2006 trafen sich die Direktoren der ZB Zürich und der UB Basel mit Fachleuten und beschlossen, die nötige Weiterentwicklung des IDS-Formats durch Pierre Gavin und das Erstellen eines Regelwerks durch eine Arbeitsgruppe gemeinsam mit anderen Bibliotheken anzugehen. Der Arbeitsgruppe sollten Christian Aliverti (ZB), Ueli Dill (UB Basel), Pierre Gavin (UB Basel), Peter Kamber (ZHB Luzern), Mikkel Mangold (UB Basel), Alexa Renggli (ZB) und Angelika Salge (ZB) angehören. Auf dem Jahrestreffen der Handschriftenbibliothekare am 08. Februar 2007 wurde der Plan kommuniziert.

Zunächst wurde in Zürich ein Regelwerk für die Katalogisierung von Briefen erarbeitet, das dringendste Desiderat aus Zürcher Sicht. In Basel wurde gleichzeitig versucht, die Übersetzung von AMREMM unter dem Arbeitstitel „KIDS Handschriften“ an die schweizerischen Gegebenheiten und Bedürfnisse anzupassen. Im August traf sich die AG dann zu einer ersten von einer ganzen Reihe von Sitzungen. Während nach AMREMM der Hauptteil des Regelwerks Handschriften galt, sollte die Brief- und Nachlasskatalogisierung in Anhängen abgehandelt werden. Folgende Prinzipien galten:

- Das neue Format muss kompatibel mit IDS-MARC sein. Es darf keine Konflikte geben, falls Druck- und Handschriften in ein- und derselben Datenbank katalogisiert werden. 
- $\quad$ Das Format soll den Export in andere Formate ermöglichen, z.B. in das durch TEI P5 (speziell Kap. 10) definierte XML-Schema, das auch von e-codices.ch angewandt wird.

- Die Katalogisierungsregeln werden an die DFG-Richtlinien Handschriftenkatalogisierung angepasst, welche auch in der Schweiz bei Handschriftenbeschreibungen befolgt werden.

- Format und Regelwerk sollen eine Katalogisierung auf verschiedenen Niveaus ermöglichen. Die Bandbreite soll von Kurzaufnahmen bis zu den detailliertesten Beschreibungen reichen. ${ }^{2}$

Im Frühjahr 2007 wurde auch eine Lösung konzipiert und realisiert, um im Verbund Basel/Bern die Sekundärliteratur zu Handschriften in DSV01 zu erfassen, zu codieren und aus den jeweiligen Handschriftendatenbanken abfragbar zu machen.

$\mathrm{Zu}$ dieser Zeit planten das Unternehmen e-codices den Aufbau einer schweizerischen Manuskript-Datenbank und der Verbund RERO die Einführung eines Archivmoduls. Im März trafen sich Vertreter von e-codices und der UB Basel, um Möglichkeiten der Zusammenarbeit zu besprechen. In der Folge nahmen zwei Vertreter der AG Handschriftenkatalogisierung an einem TEI-Workshop von e-codices teil und die UB Basel organisierte auf den 03. Juli 2008 eine kleine Tagung Elektronische Kataloge für Handschriften und Nachlässe, auf der neben Übersichten über die ausländischen Kataloge die verschiedenen Schweizer Kataloge oder Planungen dazu vorgestellt und diskutiert wurden, darunter das Archiv-Modul von RERO, Helvetic Archives der Schweizerischen Nationalbibliothek, MDb Switzerland (Swiss Manuscript Database) von e-codices und DSV05. Ich selbst machte aufgrund der bisherigen Erfahrungen mit DSV05 und der Arbeit der AG Handschriftenkatalogisierung den Vorschlag für eine zentrale Aleph-Datenbank für Nachlässe, Briefe und Handschriften:

Bestehende Datenbanken zu einem Verbund zu fusionieren ist fast nicht mehr möglich. Auf dem Gebiet der Handschriften stehen wir aber noch am Anfang. Diese Chance sollten wir nutzen und eine Verbunddatenbank einrichten:

- $\quad$ an der sich alle schweizerischen Bibliotheken beteiligen können

- in der alles Material, das in den Handschriftenabteilungen aufbewahrt wird (Papyri, Handschriften vom Mittelalter bis heute, Briefe, Autographen, hs. Musikalien, Nachlässe/Privatarchive, Curiosa usw.), katalogisiert werden kann.

Eine der beteiligten Bibliotheken würde mit dem Hosting und der Betreuung der Datenbank beauftragt, die anderen beteiligen sich anteilsmässig an den Kosten. Katalogisiert wird an den einzelnen Bibliotheken. ${ }^{3}$

2 Ueli Dill: Handschriften und Nachlässe im MARC-Format katalogisieren. Präsentation vom 03. August 2008.

3 Dill, MARC-Format (wie Anm. 2). 
Ab Oktober 2008 katalogisierte die Kantonsbibliothek Appenzell Ausserrhoden die umfangreichen Korrespondenzen der Textilhandelsfamilie Zellweger im St. Galler Pendant zu DSV05, in SGB05. Im November entschloss sich die UB Basel, ihre zahlreichen Musikautographen, die bisher im Hauptkatalog erfasst waren, in die Datenbank DSV05 zu migrieren.

Am 13. Januar 2009 trafen sich Vertreter der ZB Zürich, der Burgerbibliothek Bern und der UB Basel. Thema war die Katalogisierung und Digitalisierung der Sondersammlungen, Ausgangspunkt die Frage, wie Digitalisate von nicht-mittelalterlichen Handschriften zugänglich gemacht werden sollten. Meine schon früher vorgebrachte Idee einer zentralen Aleph-Datenbank wurde aufgenommen, nun aber ergänzt um eine Plattform nach dem Vorbild von e-rara.ch. Sie sollte für diejenigen Materialien, die für e-manuscripta.ch nicht in Frage kamen, dienen und wie e-rara.ch aus verschiedenen Katalogdatenbanken, vorzugsweise aber aus der geplanten zentralen Aleph-Datenbank gespeist werden. Ich erhielt den Auftrag, zusammen mit meinen Kollegen aus den Handschriftenabteilungen der ZB Zürich und der Burgerbibliothek, Patrick Andrist, Christoph Eggenberger, Urs Fischer und Marlis Stähli, einen Projektentwurf zu entwickeln und auf der nächsten Jahrestagung der Handschriftenbibliothekare vorzustellen.

Dies geschah und am 26. März stellten wir unter dem Titel „Manuscripta Helvetica“ das Projekt vor. Die damals gezeigte Graphik veranschaulicht die ins Auge gefasste Struktur:

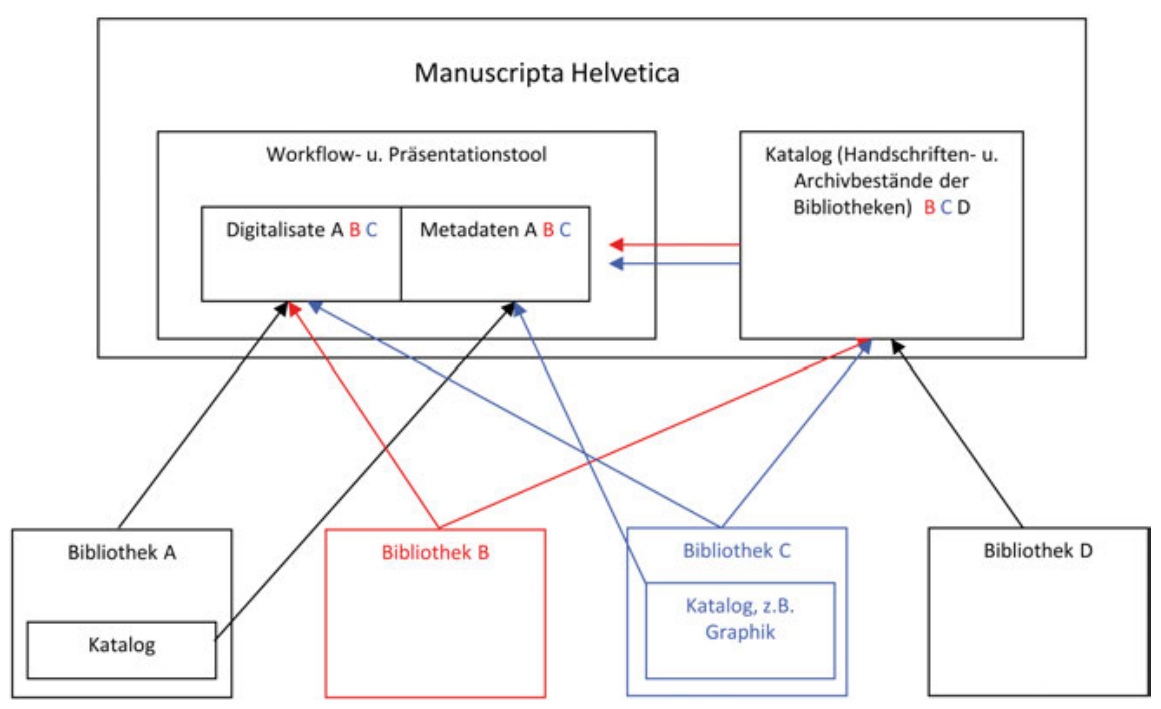

Abb. 1: am 26. März 2009 präsentiertes Modell von Manuscripta Helvetica 
Das Projekt sollte in drei Teilprojekte gegliedert werden:

- Aufbau eines Verbundkatalogs und Import bestehender Daten

- Erarbeitung eines Katalogisierungsregelwerks

- $\quad$ Einrichten eines Workflow- und Präsentationstools (in Kooperation mit e-rara) ${ }^{4}$

Die einzelnen Bibliotheken wurden aufgefordert, in einer Umfrage Stellung zum Vorschlag zu nehmen und ihre jeweiligen Bedürfnisse und Anforderungen zu formulieren. Die Diskussion war schon auf dem Jahrestreffen sehr lebhaft und wurde über den Sommer hinweg an verschiedenen Orten, u. a. auch im Leitungsausschuss von e-lib.ch, kontrovers geführt. Hauptdiskussionspunkt war das Verhältnis zu e-codices, das sich durch das Projekt, obwohl es explizit als Ergänzung, nicht als Konkurrenz konzipiert war, vor allem durch den Namen angegriffen fühlte. Um diesen Bedenken Rechnung zu tragen, verzichteten wir auf den Namen „Manuscripta Helvetica“ und ich schlug auf der Jahresversammlung des Kuratoriums Katalogisierung der mittelalterlichen und neuzeitlichen Handschriften der Schweiz am 12. Mai 2009 folgendes zeitlich gestaffeltes Vorgehen vor:

- Verbundkatalog Handschriften- und Archivbestände in der Schweiz (auf der Basis des Basler Katalogs Handschriften und Nachlässe)

- Einbindung von e-codices

- Webportal Handschriften- und Archivbestände in der Schweiz mit Präsentationstool (auf der Grundlage des Projekts e-rara)

- Metakatalog Handschriften- und Archivbestände in der Schweiz (auf der Grundlage des Projekts Swissbib) ${ }^{5}$

Aufgrund der grösstenteils positiven Rückmeldungen wurden die interessierten Institutionen zu einer Startsitzung auf den 18. September 2009 eingeladen. Die Runde erteilte mir das Mandat, einen Planungsausschuss aus Vertretern aller interessierten Bibliotheken (Handschriftenbibliothekare, IT-Fachleute und Katalogisierungsexperten) zu bilden und raschestmöglich zu einer ersten Sitzung einzuladen. Bis Ende November sollte dieser Ausschuss konkrete Vorschläge zur rechtlichen Form (inkl. Namen) und einen Zeit- und Finanzplan für das Projekt eines Verbundkatalogs erarbeiten. Ziel war es, noch vor Ende des Jahres einen

4 Ueli Dill, Patrick Andrist, Christoph Eggenberger, Marlies Stähli: Manuscripta Helvetica. Katalog und Online-Plattform für Handschriften- und Archivbestände (Handschriften des Mittelalters und der Neuzeit, Nachlässe, Briefe, Autographen, und Archivalien) aus Schweizer Bibliotheken und Archiven. Präsentation vom 26. März 2009.

5 Ueli Dill: Zur Katalogisierung und Digitalisierung von Handschriften- und Archivbeständen in der Schweiz. Sitzungsunterlage zu Traktandum 7 der Sitzung des Kuratoriums Handschriftenkatalogisierung vom 12. Mai 2009. 
Entscheid über die Schaffung eines Handschriftenverbunds und die Realisierung eines Verbundkatalogs zu fällen.

Die erste Sitzung des provisorisch „Kooperative Katalogisierung und Digitalisierung von Handschriften- und Archivbeständen“ genannten Planungsausschusses fand am 21. Oktober, eine weitere am 10. November statt. Aus mehreren Namensvorschlägen wurde dabei am Schluss „HAN - Handschriften, Archive, Nachlässe“ ausgewählt. Als Rechtsform wurde der Verein gewählt, dessen Statuten formuliert wurden. Als Betreiberin des Katalogs wurde die UB Basel bestimmt. Vorschläge für eine Fachkommission Format und Regelwerk, die sich aus Praktikerinnen und Praktikern zusammensetzen sollten, wurden zusammengestellt.

Da die ZB Zürich sich noch nicht zu einem definitiven Beitritt entschliessen konnte, beschloss man auf der Grundlage von Absichtserklärungen, HAN am 01. Januar 2010 zu starten, vorerst mit einer einjährigen Aufbauphase. Partner waren die Kantonsbibliothek Appenzell Ausserrhoden, die UB Basel, die Zentral- und Hochschulbibliothek Luzern, die Kantonsbibliothek Vadiana St. Gallen und die Zentralbibliothek Solothurn. Die wichtigsten Akteure waren in der Folge Ursula Steinegger, die von der UB Basel mit der Leitung der Regelwerksarbeiten beauftragt wurde, und Oliver Schihin, der als Systembibliothekar eingestellt wurde. Die Aufbauarbeit verlief dank der Mitarbeit aller Partner sehr konstruktiv. In verschiedenen Arbeitsgruppen wurden über mehrere Jahre hinweg die nötigen Regelwerke verfasst, bis Anfang 2014 mit dem Regelwerk für Archivbestände das letzte vorlag. Am 01. März 2011 wurde der Verbundkatalog HAN mit der Gründung des gleichnamigen Vereins offiziell aus der Taufe gehoben.

Damit war der erste Teil des ursprünglichen Konzepts realisiert, ein allen interessierten Schweizer Bibliotheken und Archiven offenstehender Verbundkatalog. Den Lead in der Realisierung des zweiten Teils, einer Plattform für Digitalisate neuzeitlicher Handschriften und Archivalien, übernahm im Herbst 2010 die ZB Zürich, die in den folgenden Jahren zusammen mit der ETH-Bibliothek und der UB Basel e-manuscripta.ch aufbaute.

\section{HAN heute}

Träger des Verbundkatalogs ist der Verein Verbundkatalog Handschriften, Archive, Nachlässe mit Sitz in Basel. Dessen Zweck ist so definiert:

1 Der Verein bezweckt, einen Verbundkatalog für den gemeinsamen elektronischen Nachweis von Handschriften, Archivbeständen und Nachlässen der Vereinsmitglieder aufzubauen und zu unterhalten. 
2 Der Verbundkatalog steht allen Sammlungen offen, die Handschriften (inkl. Musik, Karten etc.), Archivbestände und Nachlässe verwahren. ${ }^{6}$

Mitglieder des Vereins sind zurzeit die Kantonsbibliothek Appenzell Ausserrhoden, die Universitätsbibliothek Basel, die Zentral- und Hochschulbibliothek Luzern, die Kantonsbibliothek Vadiana St. Gallen und die Zentralbibliothek Solothurn. Die Direktionen dieser Institutionen treffen sich in der Regel einmal jährlich zur Generalversammlung. Die Geschäfte werden von der Verbundkoordination und dem Planungsausschuss vorbereitet.

Der Trägerverein HAN bestimmt die strategischen und normativen Grundlagen des Verbundes. Er hat die UB Basel beauftragt, den Verbundkatalog und die Verbundkoordination zu betreiben. Diese Arbeit wird von einem Verbundkoordinator (30\%), zurzeit Beat Mattmann, und einem Systembibliothekar (20\%), zurzeit Basil Marti, geleistet, dank denen der Betrieb reibungslos läuft und der Katalog sich ständig weiterentwickelt.

Heute beteiligen sich elf Bibliotheken und Archive aus sechs Kantonen am Verbundkatalog (in alphabetischer Reihenfolge):

- Appenzell Ausserrhoden: Kantonsbibliothek

- Basel: Universitätsbibliothek

- Basel: Schweizerisches Wirtschaftsarchiv

- Bern: Gosteli-Stiftung

- Bern: Universitätsbibliothek (Zentrum Historische Bestände, Archiv und Sammlung Hermann Rorschach und Schweizerische Osteuropabibliothek)

- Luzern: Zentral- und Hochschulbibliothek

- Sankt Gallen: Kantonsbibliothek Vadiana

- Sankt Gallen: Stiftsbibliothek

- Solothurn: Zentralbibliothek

- Thurgau: Kantonsbibliothek

- Zofingen: Stadtbibliothek

Ebenfalls in HAN publiziert werden seit 2017 die unter der Leitung des Kuratoriums Katalogisierung der mittelalterlichen und frühneuzeitlichen Handschriften der Schweiz erstellten Handschriftenbeschreibungen. ${ }^{7}$ Weitere Institutionen haben ihr Interesse an einem Beitritt angemeldet.

6 Statuten, revidierte Fassung vom 27. März 2017.

7 Vgl. www.codices.ch. 
Die Katalogisierung erfolgt in der Regel selbständig bei den einzelnen Verbundteilnehmern. Jede Institution nutzt dabei den Katalog in der für sie passenden Art, sei es als Verwaltungsinstrument mit Kurzaufnahmen, sei es als Möglichkeit, Sekundärliteratur zu den Sondersammlungen zu verzeichnen, sei es als Rechercheinstrument mit einer Erschliessung, die von Minimalaufnahmen bis hin zu ausführlichen Handschriftenbeschreibungen bzw. von Bestandsaufnahmen bis hin zu auf Dokumentenebene katalogisierten Archivbeständen reicht. Die Katalogisierung kann direkt in Aleph erfolgen, doch ist auch ein standardisierter Import von Erschliessungsmetadaten aus Excel-Tabellen in die Aleph-Datenbank möglich.

Die Datenbank DSV05 (bibliographische Daten) ist nach dem im Formathandbuch HANMARC für die Katalogisierung von Handschriften und Archivbeständen definierten Format konfiguriert. ${ }^{8}$ Orientierte sich dieses Format anfänglich an IDS-MARC, ist es heute, nach der Einführung der RDA im IDS, bis auf wenige Ausnahmen mit MARC21 identisch.

HAN beteiligt sich seit 2016 mit einer eigenen GND-Lokalredaktion an der Gemeinsamen Normdatenbank (GND). Die Verknüpfungen erfolgen über die IDSGND-Autoritätsdatenbank Aleph (IDS18), einen stetig aktualisierten, vollständigen Spiegel der GND in MARC21 für die Aleph-Umgebung.

Grundlage für die Katalogisierung sind die von den Verbundmitgliedern erarbeiteten Regelwerke:

- HAN-Katalogisierungsregeln für Handschriften ${ }^{9}$

- HAN-Katalogisierungsregeln für Archivbestände ${ }^{10}$

8 http://www.ub.unibas.ch/digi/a100/diverse_projekte/ubpublikationen/hanregelwerk/BAU_1 _006256804.pdf. Pierre Gavin, Bert Wessendorf, Ueli Dill, Überarbeitung (2012); Ueli Dill, Ursula Steinegger, Oliver Schihin, Überarbeitung (2014-17); Beat Mattmann, Version 19, 01. Dezember 2017.

9 http://www.ub.unibas.ch/digi/a100/diverse_projekte/ubpublikationen/hanregelwerk/BAU_1 _006258187.pdf. Erarbeitet von Ueli Dill (UB Basel), Rudolf Gamper (Vadianische Sammlung St. Gallen), Ian Holt (ZB Solothurn), Beat Mattmann (UB Basel), Peter Kamber (ZHB Luzern), Oliver Schihin (UB Basel), Ursula Steinegger (UB Basel), Monika Studer (UB Basel), Version 9, 01. Dezember 2017.

$10 \mathrm{http}: / /$ www.ub.unibas.ch/digi/a100/diverse_projekte/ubpublikationen/hanregelwerk/BAU_ 1_006255996.pdf. Erstellt von Beat Mattmann (UB Basel) und Ursula Steinegger (UB Basel) in Zusammenarbeit mit Irene Amstutz (SWA Basel), Ueli Dill (UB Basel), Wolfgang Göldi (Kantonsbibliothek Vadiana St. Gallen), Sandra Koch (ZHB Luzern) und Martin Lüpold (SWA Basel), Version 2.3, 01. Dezember 2017. 


\section{- HAN-Katalogisierungsregeln für Briefe ${ }^{11}$ \\ - HAN-Katalogisierungsregeln für Eintragungen ${ }^{12}$}

Verantwortlich für die Einhaltung der Regeln sind die einzelnen Katalogisierungsstellen, doch hat die Verbundkoordination ein Auge darauf, weist die Partner auf systematische Fehler hin und führt Massenkorrekturen aus. Vor allem aber bietet sie Beratung und Schulungen für verschiedene Levels und Gebiete an. Einmal im Jahr findet ein Verbundtreffen statt, auf dem die aktuellen Probleme diskutiert und Weiterentwicklungsvorschläge vorgebracht werden können sowie allgemein der Austausch und Wissenstransfer gefördert werden soll. Weitere Workshops werden z.B. für die Handschriftenerschliessung regelmässig durchgeführt. Alle relevanten Informationen und Dokumente sind im Wiki Babette, dem Wiki des IDS Basel/Bern, verbundweit zugänglich. ${ }^{13}$ Die Recherche in den erfassten Erschliessungsdaten und ihre Anzeige können gemeinsam oder spezifisch nach den einzelnen Partnerinstitutionen erfolgen. In Aleph selbst ermöglicht ein heute z. T. als etwas veraltet angesehener, von den Spezialisten aber geschätzter, weil an die Bedürfnisse der im Katalog erfassten Dokumententypen angepasster OPAC eine sehr präzise Recherche. Die Metadaten werden auch in hierarchischer Darstellung nach den Signaturen geordnet als Archiv- bzw. Bibliotheksplan ausgegeben oder in thematischen Zusammenstellungen präsentiert. ${ }^{14} \mathrm{Zu}$ den einzelnen Beständen können aus den Metadaten Findbücher erstellt werden, die online oder in ge-

11 http://www.ub.unibas.ch/digi/a100/diverse_projekte/ubpublikationen/hanregelwerk/BAU_ 1_006255850.pdf. Auf Grundlage der Katalogisierungsregeln für Briefmanuskripte der ZB Zürich und den Ergänzungen der KB Appenzell Ausserrhoden erarbeitet von Heidi Eisenhut, Livia Knüsel, Maya Zellweger (alle KB Appenzell Ausserrhoden), Ueli Dill, Beat Mattmann, Oliver Schihin, Ursula Steinegger (alle UB Basel), Sandra Koch (ZHB Luzern) und Alexa Renggli (ZB Zürich), Version 2.2, 01. Dezember 2017.

12 http://www.ub.unibas.ch/digi/a100/diverse_projekte/ubpublikationen/hanregelwerk/BAU_ 1_006256010.pdf. Erarbeitet von Ueli Dill (UB Basel), Sandra Frank (UB Basel), Rudolf Gamper (Vadianische Sammlung St. Gallen), Ian Holt (ZB Solothurn), Peter Kamber (ZHB Luzern), Beat Mattmann (UB Basel), Oliver Schihin (UB Basel), Ursula Steinegger (UB Basel), Version 2.2, 01. Dezember 2017.

13 http://www.ub.unibas.ch/babette/index.php/Hauptseite (Zugang nur für Verbundmitglieder). 14 Dabei kann der Archivplan angezeigt werden (alle HAN-Partner: http://www.ub.unibas.ch/h an/suche-in-han/hierarchische-gliederung/, eine einzelne Institution: https://www.gosteli-fou ndation.ch/de/recherche/hierarchische-suche) oder es kann eine andere Anzeige eingerichtet werden (Nachlässe der UB Basel alphabetisch geordnet: http://www.ub.unibas.ch/ibb/api/ub nachlass/personen.html, die systematische Gliederung der über 500 Firmen- und Verbandsarchive sowie Personennachlässe des Schweizerischen Wirtschaftsarchivs: http://www.ub.unibas. ch/ub-wirtschaft-swa/schweiz-wirtschaftsarchiv/privatarchive/). 
druckter Form in den Sonderlesesälen zugänglich sind. Erklärtes Ziel des Verbunds ist es, die Metadaten, soweit sinnvoll, in passende Metakataloge zu exportieren bzw. von diesen ,harvesten` zu lassen. Zurzeit sind alle Metadaten oder eine von den Dateneigentümern bestimmte Auswahl in swissbib, swissbib Basel Bern, im Nachlassportal Kalliope und in Archives online abfragbar. Diese Exporte haben die Benutzungszahlen sofort merkbar wachsen lassen.

Selbstverständlich dient HAN auch als Ausgangspunkt für Verlinkungen zu allen anderen relevanten Informationen der jeweiligen Dokumente: zu Repertorien wie dem Handschriftencensus, zu online zugänglichen Katalogen, Findbüchern und Beschreibungen sowie $\mathrm{zu}$ virtuellen Handschriftenbibliotheken wie e-codices oder e-manuscripta.ch.

Den Verbundpartnern bietet die Universitätsbibliothek Basel als Dienstleistung auf www.e-manuscripta.ch auch die Digitalisierung und Aufschaltung von Medien an.

\section{Vom Nutzen und Nachteil von HAN für die Verbundpartner und die Benutzerschaft}

Nutzen und Nachteile von HAN ergeben sich aus den Grundpfeilern von HAN:

- HAN ist ein Verbundkatalog, realisiert mit einem mächtigen, allerdings mittlerweile in die Jahre gekommenen Bibliothekssystem.

- HAN umfasst Handschriften- und Archivbestände und versucht, bibliothekarische und archivarische Traditionen zu verbinden.

- HAN ist konsequent nach internationalen und nationalen Normen ausgerichtet.

- HAN erlaubt verschiedene Katalogisierungsniveaus.

Als gut gepflegter Verbundkatalog garantiert HAN im Vergleich zu Einzelkatalogen und Metakataloglösungen für die Verbundpartner:

- bessere und konsistentere Daten

- $\quad$ eine grössere Sichtbarkeit der Bestände in HAN selbst, vor allem aber auch durch die gemeinsam exportierten Daten in andere Kataloge

- geringere Kosten

- sofortige Publikation der eingegebenen Metadaten, wenn gewünscht

- höhere Performanz und Stabilität

- eine besser gesicherte Nachhaltigkeit

- Anschluss an neue Entwicklungen

- Vereinfachung künftiger Migrationen 
- Auslagerung des Systemunterhalts

- Support und Ausbildung der Katalogisierenden

- engen und institutionalisierten Kontakt und Austausch mit anderen Institutionen

Der Vereinheitlichungszwang in Verbundkatalogen verunmöglicht oft, dass den individuellen Bedürfnissen der einzelnen Verbundpartner entsprochen werden kann. Das ist auch bei HAN teilweise so, doch lassen sich dank der Überschaubarkeit und der Innovationsfreude des Verbunds in der Regel befriedigende Lösungen finden.

Für die Forschenden hat der Aleph-Verbundkatalog folgende Vorteile:

- Recherche in den Beständen mehrerer Bibliotheken von einem Zugriffspunkt aus

- HAN-Daten sind auch in grossen Metakatalogen wie Kalliope, swissbib und Archives online abfragbar

- sehr präzise und detaillierte Abfragemöglichkeiten im OPAC, auch für kodikologische Fragestellungen wie Einbandstempel, für frühere Besitzer etc.

Dass HAN sowohl Handschriften wie Archivbestände umfasst, passt zu den Sammlungen in Universitäts- und Kantonsbibliotheken, wo diese Dokumente oft zusammen aufbewahrt werden und ineinander übergehen. Für die Bearbeitenden wie auch für die Benutzenden ergeben sich durch den gemeinsamen Katalog Synergieeffekte. Was die Recherche betrifft, ist HAN einem Archivsystem überlegen; hingegen ist die Erfassung von Archivbeständen für den Bearbeiter etwas weniger komfortabel als in den gängigen Archivsystemen.

Nur Vorteile hat die konsequente Ausrichtung auf internationale Normen für alle Beteiligten. Sie erleichtert den neuen und ungeübten Benutzenden die Orientierung. Sie erlaubt es, die fein granulierten Daten in wissenschaftliche Kontexte unterschiedlichster Art zu exportieren. So wird zurzeit eine Kooperation mit dem Forschungsprojekt Theologenbriefwechsel im Südwesten des Reichs in der Frühen Neuzeit (1550-1620) der Heidelberger Akademie der Wissenschaften vorbereitet mit dem Ziel, dass unsere einschlägigen Daten übernommen und angereichert zurückgegeben werden. Vor allem aber ist die Anwendung internationaler Normen im Hinblick auf spätere Migrationen eine ,conditio sine qua non' für die Nachhaltigkeit des Katalogs.

Vor allem für die Katalogisierungsstellen wichtig ist das System der unterschiedlich definierten Katalogisierungsniveaus. Dies erlaubt die dringend nötige Flexibilität bei der Rekatalogisierung. Die Neukatalogisierung kann dadurch, genau dokumentiert, an die Erschliessungsressourcen und die Bedeutung des Materials angepasst werden. 
Die Erfahrung der letzten Jahre zeigt, dass HAN in seiner kurzen Geschichte dank seines Innovationsgeistes und seiner Flexibilität den allgemeinen Entwicklungen immer ein paar Schritte voraus war. So hat sich HAN an MARC21 angelehnt, bevor dieses Format im IDS mit der Einführung der RDA zur Anwendung kam. Dasselbe gilt für die GND, mit der HAN zwar nicht verknüpft war, der es aber in den Ansetzungen, auch mit der Angabe der Lebensdaten, schon länger folgte. So kam es, dass die Einführung der GND im IDS für die HAN-Partner viel einfacher war als für die übrigen Verbünde und sich heute prozentual viel weniger nicht-GND-konforme Eintragungen in HAN finden.

\section{HAN in der Zukunft}

In den vergangenen Jahren wurde an der UB Basel unter dem Arbeitstitel „swissbib historic“ ein Konzept für einen nationalen Katalog der historischen Bestände in der Schweiz erarbeitet. Er war als Spezialview von swissbib geplant. In ihm sollten alle elektronisch zugänglichen Metadaten zum schriftlichen Kulturerbe der Schweiz versammelt werden, also auch zu den alten Drucken. Spezielles Augenmerk wurde dabei auf die Discovery-Ebene gelegt. Sie sollte die speziellen Bedürfnisse der Forschenden, die mit historischen Materialien arbeiten und heute oft nicht angemessen bedient werden, abdecken. Mit swissbib historic würde der letzte Teil des ursprünglichen Konzepts umgesetzt, ein Metakatalog Handschriften- und Archivbestände in der Schweiz.

Diese Materialien dienen der Forschung als Quelle, sind aber gleichzeitig oft auch Forschungsgegenstand. Ihr Umfang ist, verglichen mit den modernen Beständen, klein aber fein. Auch die Zahl der Benutzenden ist eher gering. Im Gegensatz dazu ist der Erschliessungsaufwand hoch, sind diese Dokumente doch sehr vielfältig, nicht standardisiert und von ihrer langen Geschichte geformt. Um den Forschenden den Zugang dazu zu ebnen, müssen vergleichsweise grosse Erschliessungsanstrengungen unternommen werden. Sie lohnen sich aber, denn oft sind diese Bestände Zimelien und Alleinstellungsmerkmale der betreffenden Institutionen.

Nachdem die Idee zur Swiss Library Service Platform (SLSP) geboren wurde und sich abzeichnete, dass sie auch verwirklicht wird, beschlossen wir, die Entwicklung von swissbib historic vorläufig $\mathrm{zu}$ sistieren, da sie sinnvollerweise im grösseren Rahmen von SLSP realisiert werden müsste.

Was bedeutet das für die Zukunft von HAN? Wie kann, wie wird HAN sich weiterentwickeln? Die einzig sinnvolle Antwort auf diese Frage lautet: im Rahmen von SLSP. Deshalb haben die HAN-Verbundpartner, aber auch andere Biblio- 
theken als Anforderung an SLSP auch die Verwaltung des historischen Bestands angemeldet.

Wird eine Integration von HAN in SLSP möglich, wird dies für HAN wie für die anderen integrierten Verbünde die Auflösung bedeuten, nicht aber das Ende. Einerseits wird die Idee von HAN, als nationaler Katalog zu dienen, ihrer Verwirklichung näherkommen. Anderseits werden die Erfahrungen von HAN hoffentlich in SLSP einfliessen. Ich sage hoffentlich, denn ein Hauptmerkmal von HAN wird vermutlich mindestens teilweise fallen, nämlich dass es ein Sonderkatalog ist; ein Sonderkatalog zwar, der verschiedenste Sondermaterialien zusammenfasst, der aber als alleinstehender Katalog für diese Sondermaterialien und ihre Benutzenden die günstigsten Bedingungen schaffen kann. Diese Idee stand ganz am Anfang und hat sich bewährt. Nur so konnte erreicht werden, was erreicht wurde.

Im Riesenkomplex SLSP werden die Katalogisate von HAN und von vergleichbaren Katalogen einen kleinen Anteil am Ganzen bilden. Die Herausforderung bzw. Pflicht für die treibenden Kräfte hinter SLSP wird es sein, diesem kleinen Teil die nötige Aufmerksamkeit zukommen zu lassen. Zwar sind die Sonderbestände und ihre Benutzenden zahlenmässig klein, dafür aber für das Renommee wichtig und in einer immer stärker vereinheitlichten und zentralisierten Bibliothekswelt immer bedeutsamer. Ihre Integration sollte deshalb nicht auf die lange Bank geschoben werden. HAN als grösster schweizerischer Verbundkatalog für Handschriften- und Archivbestände kann dabei mit den nötigen Adaptionen als Vorbild und Ideengeber dienen, was die Organisation der Verbundarbeit, das Format und die Regelwerke angeht. HAN kann übertroffen werden, wenn die unter dem Titel „swissbib historic“ formulierten Anforderungen an die Discovery für alle historischen Bestände, handschriftliche wie gedruckte, realisiert werden und damit auch eine noch engere Verzahnung mit den Digitalisierungsplattformen erreicht wird.

Die an sich wünschenswerte Einbindung der Archivkataloge wird, wenn überhaupt, erst mittelfristig in grösserem Umfang möglich werden. Umso wichtiger ist es, auch in Zukunft am HAN-Prinzip festzuhalten, das jeweils Beste aus der Erschliessungstheorie und -praxis sowohl im Archiv- wie auch im Bibliotheksbereich zu vereinigen. Dies bedingt inhaltlich eine Weiterentwicklung mit Blick auch auf die Bestrebungen im Archivwesen und weiterhin eine Grundeinstellung der ausgestreckten Hand zu den Archiven.

Eine Herausforderung wird die nötige Umstellung auf RDA sein. Wir bereiten uns darauf vor, indem wir Vertretungen in die entsprechenden RDA-Arbeitsgruppen der Deutschen Nationalbibliothek delegiert haben.

Eine weitere Herausforderung werden Datenschutzfragen werden. In HAN sind rezente Nachlässe durchaus datenschutzkonform oft recht tief erschlossen dies zum Vorteil der Benutzenden, manchmal aber auch zur Irritation noch 
lebender Verfasser, Adressaten oder anderswie Beteiligter. In einem Archivzusammenhang, d.h. im OPAC von HAN selbst oder auch in Kalliope, stört das in der Regel kaum. Werden die Daten aber, wie es bei HAN via swissbib schon praktiziert wird und bei SLSP die Regel wäre, zusammen mit Bibliotheksdaten angeboten, kann dies zu Problemen führen. Entscheidend wird sein, die in einer Datenbank vereinigten Katalogisate in der Discovery zu trennen, um Frustrationen bei den nur an Bibliotheksdaten Interessierten und Ärger bei den in Archivkatalogisaten Vorkommenden zu vermeiden. Je grösser die Datenbank und der Benutzerkreis, umso spezifischer müssen die Abfragemöglichkeiten gestaltet werden.

Die grösste Herausforderung der nächsten zehn Jahre, ob in HAN oder anderswo, wird es aber sein, alle in den Spezialsammlungen im Laufe des letzten Jahrhunderts angehäuften analogen Erschliessungsdaten in die digitale Welt zu überführen. Dies ist ein überaus umfangreiches Vorhaben, nicht zu vergleichen mit der auch schon aufwendigen Rekatalogisierung der Druckbestände, da die vorhandenen Daten sehr vielgestaltig sind. Trotzdem ist sie unbedingt nötig, damit das, was in der analogen Welt zurückbleibt, nicht aus dem Fokus der Forschung verschwindet. Ein Nachfolgesystem von HAN, sei es SLSP oder etwas Anderes, muss es wie HAN möglich machen, diese vielfältigen Daten fein strukturiert auf verschiedenen Katalogisierungsniveaus in die Zukunft $\mathrm{zu}$ bringen. Anders als bei modernen Medien ergeben sich bei der Katalogisierung von historischen Dokumenten, die als Handschriften ,eo ipso“ unikal sind und als Druckschriften durch ihr Alter unikal werden, auch in grossen elektronischen Katalogen kaum Synergien. Und auch wenn die grosse Aufgabe der Rekatalogisierung bewältigt sein wird, bleibt die Katalogisierung eine nie ganz abgeschlossene Aufgabe. Denn das Wissen über die historischen Dokumente wächst dank der Forschung ständig und muss in passender Form im Katalog festgehalten werden.

Zum Schluss ein Wort an die Bibliotheksdirektionen: Kataloge für die historischen Bestände sind schöne, aber zarte Pflänzchen. Sie verlangen Schutz und Aufmerksamkeit. Das Rezept zum Erfolg haben sie mit dem am Anfang erwähnten HAN Mongolian Barbecue gemeinsam: die Kombination von Grundzutaten hoher Qualität mit einer Vielfalt individuell wählbarer Zutaten, so dass sowohl alle angeschlossenen Institutionen als auch die Benutzenden und Forschenden das bekommen, was ihren Bedürfnissen entspricht. 